\title{
Dysphonia, a Manifestation of a Rare Adverse Effect of Inhaled Corticosteroids
}

\author{
Miguel Mayo-Yáñez ${ }^{1,2^{*}}$ and Irma Cabo-Varela ${ }^{1}$ \\ ${ }^{1}$ Otorhinolaryngology - Head and Neck Surgery Department, Complexo Hospitalario Universitario A \\ Coruña (CHUAC), 15006, A Coruña, Galicia, Spain. \\ ${ }^{2}$ Clinical Research in Medicine, International Center for Doctorate and Advanced Studies (CIEDUS), \\ Universidade de Santiago de Compostela (USC), 15782, Santiago de Compostela, Galicia, Spain.
}

A 76-year-old woman with fluctuating dysphonia, accompanied by heartburn and a need of continuous throat clearing, of a month of evolution. As personal history she presents hypertension, type II diabetes, dyslipidemia and bronchial asthma. Non-smoker or alcohol consumer.

The patient did not present fever, the blood analysis showed no alterations and there were no changes in the symptomatology with the corporal posture. She reported worsening during the day and having been treated with Proton-pump inhibitors without improvement.

The otorhinolaryngological exploration was strictly normal except for the nasofibroscopy, finding minimally elevated leukoplastic lesions at subglottic level accompanied by increased vascularization in the vocal cords, with minimal edema and hyperemia (Figure 1).

Given the symptoms and history of the patient, a single course with Fluconazole was established, resulting effective and causing the complete disappearance of the lesions and

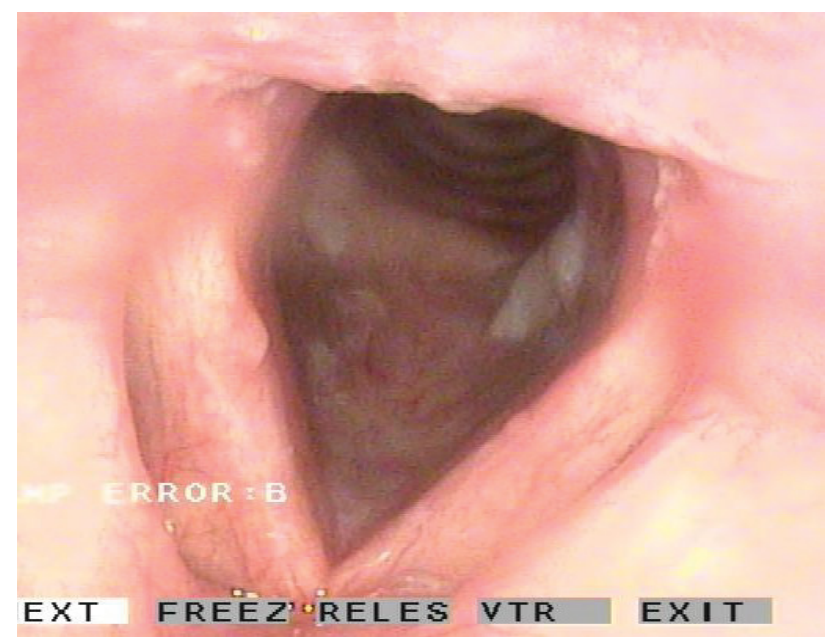

Figure 1: Bilateral leukoplastic lesions at subglottic level accompanied by increased vascularization in the vocal cords, with minimal edema and hyperaemia. symptoms, and confirming the diagnosis of suspicion: Subglottic candidiasis.

On one hand, inhaled corticosteroids are the mainstay treatment for all severities of reactive airway disease, although their systemic side-effect profile has been well studied, the potential oropharyngeal and laryngeal adverse effects are often under recognized. On the other hand, when host immune mechanisms and the protective mucosal barrier are impaired, Candida overgrowth can lead to infections of the larynx. Predisposing factors for laryngeal candidiasis include immunodeficiency syndromes, immunosuppressive medications, nutritional deficiencies, diabetes, previous antibiotic therapy, inhaled corticosteroid therapy [1], radiation therapy, smoking, reflux disease, trauma, and chemical or thermal injury $[2,3]$.

Only case reports and three case series of laryngeal candidiasis have been published in the literature, being the isolated subglottic location very rare. The diagnosis is clinical and the most commonly presented symptom is dysphonia (60-70\%). followed in decreasing order by chronic cough, shortness of breath, sore throat, globus sensation and even hemoptysis. Endoscopic examination of the larynx reveals a characteristic white or grey pseudomembrane, although some patients have erythema alone. Mucosal edema and ulceration can also be present. This set of signs and symptoms can be confused with other pathologies, simulating squamous cell carcinoma or a precancerous lesion, which could result in biopsy. This management should be reserved for cases in which injuries persist despite adequate treatment in order to avoid possible

*Corresponding author: Miguel Mayo-Yáñez, Otorhinolaryngology - Head and Neck Surgery Department, Complexo Hospitalario Universitario A Coruña (CHUAC). As Xubias 84, 15006, A Coruña, Spain.

Accepted: November 10, 2020

Published online: November 12, 2020

Citation: Mayo-Yáñez M, Cabo-Varela I (2020) Dysphonia, a Manifestation of a Rare Adverse Effect of Inhaled Corticosteroids. Arch Immunol 2(1):24-25

Copyright: (C) 2020 Mayo-Yáñez M, et al. This is an open-access article distributed under the terms of the Creative Commons Attribution License, which permits unrestricted use, distribution, and reproduction in any medium, provided the original author and source are credited. 
complications, including vocal cord scarring and general anaesthetic risks [2,3].

The treatment of choice is a single course of antifungal medication, being fluconazole highly effective eradicating Candida, and often accompanied by elimination of predisposing factors, vocal hygiene, eliminating gastroesophageal reflux, and increasing hydration. In certain cases, voice therapy is necessary, either during or after primary treatment. Early recognition and treatment of this disease is important to prevent the spread of infection and the accomplishment of unnecessary complementary procedures $[2,3]$.

\section{Disclosures}

There was no conflict of interest and authors have noth- ing to declare. This research involved human participants and was approved by the Hospital's Ethics Committee. Informed consent was obtained from all individual participants included in the study.

\section{References}

1. Chmielewska M, Akst LM. (2015) Dysphonia associated with the use of inhaled corticosteroids. Curr Opin Otolaryngol Head Neck Surg 23: 255-259.

2. Wong KK, Pace-Asciak P, Wu B, et al. (2009) Laryngeal candidiasis in the outpatient setting. J Otolaryngol Head Neck Surg 38: 624-627.

3. Neuenschwander MC, Cooney A, Spiegel JR, et al. (2001) Laryngeal candidiasis. Ear Nose Throat J 80: 138-139. 